\title{
MS35-P13 | EXPLORING THE LIMITS OF IOdONIUM (I+) FORMATION
}

Ward, Jas (University of Jyväskylä, Jyväskylä)

The occurrence of halonium ions (halogen atoms in the 1+ oxidation state) has been known for some time and exist as linear two-coordinate complexes stabilised by appropriate nucleophilic ligands. Halonium species can be straightforwardly synthesised by cation exchange of the analogous $\mathrm{Ag}(\mathrm{I})$ complex with an elemental halogen ( $\mathrm{Br}_{2}$, $\mathrm{I}_{2}$ ), though as the reactivity increases as you go up group 17 , examples of lodonium $\left(\mathrm{I}^{+}\right)$complexes in the solid-state are most prevalent. However, given their intrinsic reactivity, with species like $\left.\left[\mathrm{I}_{(\mathrm{pyridine}}\right)_{2}\right]\left[\mathrm{BF}_{4}\right]$ even being sold commercially as reagents, the number of halonium complexes studied in the solid-state is still quite small. Studies investigating the links between halonium formation, steric considerations of the nucleophilic ligands, or the identity of the counterion are also largely absent in the literature. Understanding the conditions which favour/disfavour halonium formation would greatly aid in the future development of this area of research.

Herein we report the solid-state structures of two-coordinate $\mathrm{Ag}(\mathrm{I})$ complexes possessing a range of different common counterions, all of which were synthesised for their potential to be converted into the analogous halonium species. Those successfully converted into the analogous iodonium complexes, and the reasons thereof, will also be discussed. 